\title{
Lo que nos enseña el 27F en Chile sobre el impacto de un desastre natural en la salud infantil
}

\author{
MIGUEL A. CORDERO V. ${ }^{1}$, PAULA B. REPETTO L. ${ }^{2}$, MARY CATHERINE ARBOUR ${ }^{3}$ \\ 1. School of Social and Community Medicine, University of Bristol, UK. \\ 2. Escuela de Psicología, Pontificia Universidad Católica de Chile. National Research Center for Integrated Natural Disasters \\ Management. \\ 3. Center on the Developing Child, Harvard University. Division of Global Health Equity of Brigham and Women's Hospital, \\ Boston USA..
}

\begin{abstract}
Lessons learned from the $27 \mathrm{~F}$ on the impact of a natural disaster on child health

There is a large scientific literature about disasters' effects on adult population; however, comparatively few studies explore the effects of natural disasters on children, especially during early childhood and school years. Three years after the Chilean earthquake of 27 February 2010, we review the national and international literature on natural disasters and their effects on children. We highlight advances and identify knowledge gaps, in particular contributions and limitations of national research about the impacts of the 2010 Chilean earthquake in child health. In the three years since the Chilean earthquake, post-disaster child health research has made important advances toward better understanding of the impact of disasters on children's mental health and overall well-being. The articles included in this edition of the Revista Chilena de Pediatría represent an important contribution to a nascent literature.
\end{abstract}

(Key words: Natural disaster, earthquake, 27F, mental health).

Rev Chil Pediatr 2013; 84 (1): 10-19

\section{RESUMEN}

Existe una amplia base de literatura científica sobre los efectos de los desastres en la población adulta, sin embargo, en comparación hay pocos estudios que exploren los efectos de los desastres naturales en los niños, especialmente durante la primera infancia y los años escolares. Transcurridos 3 años del terremoto del 27 de febrero de 2010 en Chile, se revisa la literatura sobre los desastres naturales y sus efectos sobre los niños a nivel nacional e internacional. Se resaltan los progresos y se identifican las brechas de conocimiento, los aportes y limitaciones de la investigación nacional sobre los impactos en la salud infantil del terremoto de 2010 en Chile. Se concluye que la investigación en salud infantil ha logrado importantes avances hacia una mejor comprensión del impacto de los desastres en la salud mental y el bienestar general de los niños. Los artículos incluidos en este número de la Revista Chilena Pediatría representan una importante contribución a la naciente literatura. (Palabras clave: Desastre natural, terremoto, 27F, salud mental).

Rev Chil Pediatr 2013; 84 (1): 10-19

Recibido el 15 de enero de 2013, devuelto para corregir el 24 de enero de 2013, segunda versión el 30 de enero de 2013 , aceptado para publicación el 10 de febrero de 2013 .. 


\section{Antecedentes}

El saber popular en Chile recuerda que a cada generación "le toca" un gran terremoto, uno de características catastróficas, con decenas o cientos de muertos, heridos, daños masivos en la infraestructura, y del cual usualmente el país demora varios años en reponerse ${ }^{1}$. De hecho Chile es considerado uno de los lugares de mayor sismicidad del mundo ${ }^{2}$. A lo largo de la historia se han registrado muchos terremotos y se estima que en el Siglo XX y lo que llevamos el Siglo XXI, el territorio nacional ha estado expuesto a al menos 75 terremotos de magnitud 7 o mayor.

El 27 febrero de 2010 Chile fue impactado por un terremoto de gran magnitud $(8.8$ Richter), con un epicentro a 117 kilómetros al norte de la ciudad de Concepción, afectando a seis de las quince regiones del país. Este terremoto es el segundo de mayor dimensión que se ha registrado en el país y uno de los 10 más grandes que tenga registro la historia moderna ${ }^{3}$. Alrededor de 30 minutos después del movimiento principal, violentas olas inundaron y arrasaron con vastas zonas de la costa de la VII y VIII Región. Más de dos millones de personas se vieron afectadas directamente por el terremoto principal y sus consecuencias. Alrededor del 11\% de las viviendas (370 mil) fueron dañadas o destruidas. La fuerza laboral también se vio afectada, se perdieron más de 67 mil puestos de trabajo y se estima que las $^{2}$ pérdidas económicas fueron de 30 billones de dólares ${ }^{3}$.

La Encuesta Nacional de Caracterización Socioeconómica (CASEN 2010) implementada inmediatamente luego del terremoto, mostró mayores efectos en los hogares de más bajos ingresos; "En las seis regiones afectadas el $12 \%$ de las personas del quintil más pobre experimentaron daño mayor o destrucción de la vivienda, comparado con un $4,6 \%$ en el quintil más rico", ante lo cual el informe concluye: “(...) El desastre deja en evidencia que los pobres no sólo tienen escasos ingresos, sino que están más afectos a experimentar los efectos adversos de los desastres naturales"

Millones de niños chilenos sobrevivieron a este terremoto, miles fueron testigos de la destrucción de sus hogares, escuelas y comunidades, experimentando cambios drásticos en su vida cotidiana. Producto de la pérdida de viviendas, 4.350 familias fueron emplazadas en 106 asentamientos habitacionales de emergencia denominados Aldeas. Cerca de dos mil niños y niñas menores de seis años de edad se trasladaron con su familia a vivir en estos campamentos, equipados con unidades de vivienda de emergencia y servicios básicos de saneamiento ${ }^{6}$.

Al cumplirse el tercer aniversario del terremoto y tsunami del 27 de febrero del año 2010, es importante revisar la evidencia científica sobre las consecuencias de la exposición a desastres naturales en niños y considerar qué hemos aprendido de este terremoto en particular. Muchas lecciones deben tomarse, a partir no sólo de la respuesta del país en términos físicos, de infraestructura, organizaciones y personas, sino que también considerando los impactos sanitarios que ha tenido este evento en las vidas de muchos niños y sus familias, y que continuará teniendo por muchos años más.

El objetivo de este artículo es realizar una revisión narrativa de la literatura respecto de las consecuencias en la salud mental, de la exposición a desastres naturales en la infancia, y los factores que se reconoce influyen en estos resultados. Al hacer la revisión incluimos los resultados de estudios realizados en Chile y discutimos las implicancias que pueden tener para la población infantil dados algunos antecedentes de la literatura internacional. Esperamos que este trabajo contribuya a comprender mejor el problema y a reconocer las necesidades particulares de niños y niñas chilenos cuando se enfrenten a los desastres naturales que seguro habrá en el futuro.

\section{Revisión de la literatura}

Históricamente, los niños han sido una población poco estudiada frente a desastres naturales. Sin embargo, en las últimas dos décadas varios estudios en diversos eventos tales como terremotos, tsunamis, o grandes accidentes, han demostrado que los niños se encuentran entre las personas más vulnerables a los efectos negativos de los desastres ${ }^{7-10}$. 
Los investigadores han observado que los niños que sobreviven a los desastres están en mayor riesgo de desarrollar diversos problemas de adaptación psicológica y del comportamiento, incluido los trastornos de la salud mental. Aunque no hay un claro acuerdo sobre la magnitud y extensión de estos efectos en el tiempo, las prevalencias esperadas en las semanas, meses y años posteriores son alrededor de un $17 \%$ en síntomas de ansiedad, 31\% depresión, y 50-60\% de síntomas de Trastorno por Estrés Postraumático (TEPT) ${ }^{10-15}$

La severidad de los síntomas psicológicos se ha relacionado con la exposición al desastre y las experiencias traumáticas resultado de este; incluyendo la pérdida del hogar, el desplazamiento, las inundaciones, el riesgo de perder la vida o ser testigo de la muerte de alguien. Asimismo una historia de experiencias traumáticas anteriores y tener otros antecedentes previos de problemas de salud mental, pueden poner a los niños y adultos aún en más riesgo de problemas en su salud mental ${ }^{12,16}$. Algunos estudios también muestran que las niñas quienes están más en riesgo de presentar problemas de salud mental ${ }^{14,17,18}$. Consistente con estos resultados, en un estudio realizado con una muestra de 304 adolescentes de la ciudad de Chillán y que recolectó la información entre septiembre y octubre de 2010, se detectó una alta prevalencia de síntomas TEPT, depresión $\mathrm{y}$ ansiedad ${ }^{19}$. Estos autores también encontraron una dramática diferencia entre hombres y mujeres, mostrando más de un $30 \%$ de mujeres con síntomas y de mayor intensidad. Similares resultados son reportados en el estudio de Briceño y cols (2013, en este número) en el cual participaron 89 niños entre tercero y sexto básico de la región del Maule que fueron evaluados 8 meses después del terremoto. Los resultados muestran que un $40,4 \%$ de los niños presentaban síntomas de TEPT y que ese porcentaje era más del doble en mujeres que en varones ${ }^{20}$. En el estudio de Cova y cols (2013, también en este número), que incluyó una muestra de 1.300 niños entre 9 y 16 años de la región del BioBío, los investigadores reportaron una prevalencia de síntomas TEPT de $30,4 \%$ en niñas y de $15 \%$ en niños ${ }^{21}$.

A pesar de los avances en la comprensión de los efectos de los desastres en la salud mental de los niños, hay una serie de lagunas en la investigación sobre el bienestar de los niños y los procesos de adaptación posterior a desastres.

Weissbecker y cols, describen que los niños después de un desastre pueden experimentar "una serie de eventos estresantes en cascada, que puede durar meses o incluso años" 22 . A veces estos tienen que ver con las pérdidas materiales, humanas, el desplazamiento y las reacciones emocionales de sus propios cuidadores $^{23}$. Por esta razón, se ha propuesto que la investigación debe tener en cuenta las múltiples dimensiones del desastre, así como las respuestas de estrés psicológico y fisiológico inmediato. Se debe considerar tanto las experiencias estresantes relacionadas con el desastre mismo, como aquellas derivadas y las que son parte de la historia del niño, debido al efecto acumulativo de exposiciones sucesivas a experiencias estresantes ${ }^{24,25}$. Se ha reconocido que la exposición a estresores cotidianos no directamente relacionados con el desastre, tales como la pobreza, la pérdida de la autonomía y el apoyo social debilitado, pueden predecir mejor los resultados que las experiencias derivadas del desastre en específico ${ }^{26-29}$.

Por otra parte, los estudios que tienen en cuenta los resultados no psicológicos, tales como la seguridad alimentaria, la estabilidad del cuidador, el acceso a servicios de salud y educación, y otros, permitirían comprender mejor los mecanismos a través de los cuales la exposición a los desastres ejerce su impacto en la población infantil. Estos resultados son especialmente importantes de reconocer en países en desarrollo. En Chile varios estudios epidemiológicos muestran una alta prevalencia de trastornos de salud mental en adultos ${ }^{30-32}$, alta exposición a violencia intrafamiliar y una mayor incidencia de pobreza en familias con niños ${ }^{33}$, todas condiciones reconocidas como de riesgo para trastornos de salud mental. De hecho, se ha propuesto que personas, niños y adultos, expuestas a desastres en países en desarrollo, pueden presentar más sintomatología comparado con personas en países desarrollados $^{34}$. Por ejemplo, un estudio realizado en Chile después del terremoto del 85, mostró una 
prevalencia de TEPT mucho más alta comparado con eventos similares en otros lugares del mundo ${ }^{35}$.

Una segunda brecha en la literatura sobre desastres, se relaciona con los efectos en los niños pequeños. Muchos investigadores han planteado la hipótesis de que los niños pequeños pueden estar más vulnerables a los efectos de los desastres, ya que son más dependientes de los adultos. Al mismo tiempo, en este período las personas son más vulnerables a la exposición a estrés tóxico, con consecuencias a largo plazo para la salud y el funcionamiento adaptativo. Por otra parte, debido a las limitaciones del desarrollo cognitivo, los niños no pueden comprender de manera cabal el evento y sus consecuencias y tienen un repertorio conductual más limitado, lo que no les permite enfrentar de manera efectiva el evento ${ }^{36-38}$. Las limitaciones metodológicas para evaluar a esta población es un problema difícil de abordar por los investigadores. En los estudios realizados se han utilizado una gran variedad de escalas y estrategias de medición que hacen los resultados difíciles de comparar ${ }^{27}$. Por esta razón, no sorprende que los resultados de estudios con niños en edad preescolar y escolar sean diversos, mientras algunos muestran que los otros más pequeños presentan mayores problemas ${ }^{39,40}$.

Así mismo, recientemente se ha puesto en cuestionamiento mucho de los resultados provenientes de estudios tranversales ${ }^{39}$, basados en encuestas de síntomas y cuestionarios de autoreporte. Estos estudios podrían sobreestimar o subestimar, dependiendo del caso, la prevalencia de fenómenos como el Trastorno por Estrés Postraumático. En tal sentido, Bonanno y cols ${ }^{41}$, en una reciente revisión de la literatura, sugieren que existe un amplio rango de reacciones frente a estos eventos y que, si bien, los síntomas que las personas presentan son del espectro del estrés postraumático, es importante evaluar otros trastornos y reacciones, como la depresión, ansiedad, síntomas conductuales e incluso abuso de sustancias en adolescentes. La variedad de reacciones es más amplia y es esencial reconocer que la norma es presentar una diversidad importante de síntomas y no sólo un tipo de problemas.
Análisis longitudinales de la trayectoria de respuestas en desastres de diverso tipo, muestran que lo normativo es una respuesta adaptativa, sugiriendo que lo común es entonces la resistencia frente al estrés y no la patología ${ }^{39}$. Las investigaciones deberían abordar los factores que predicen estas distintas trayectorias y por lo tanto el diseño de los estudios prospectivos, longitudinales, para permitir una visión más ajustada a la realidad que el sólo hecho de mediciones transversales. Muchos niños son muy resistentes al estrés y muchos más pueden desarrollar reacciones "normales" de adaptación que se presentan por períodos más limitados, y otros pueden desarrollar problemas evidentes, que a veces se manifiestan en trastornos mentales evaluables sólo en el largo plazo.

Una tercera limitación de la literatura tiene que ver con el desarrollo de intervenciones efectivas. En 2007 varios organismos internacionales publicaron en conjunto, un documento guía para el desarrollo de intervenciones en situación de emergencia y desastre, basado en la investigación y la experiencia internacional ${ }^{42}$. En este se señala como tarea esencial promover actividades que contribuyan a volver lo más pronto posible a las actividades de la vida cotidiana del niño y las familias en general, que las intervenciones deben ser implementadas por personas que conocen a los niños y que se evite usar consejería de trauma como primera estrategia de intervención. En este documento se reconoce el rol que juegan los padres, los centros de salud de atención primaria y las escuelas para apoyar a los niños y la necesidad de entregarles herramientas para que puedan contribuir con este objetivo. Los tratamientos de orientación conductual cognitiva deben ser implementados en etapas posteriores y con niños que presentan sintomatología más estable ${ }^{43}$. En esta misma línea, en una revisión de las estrategias de intervención realizada por LaGreca y Silverman (2009), las autoras concluyen que la evidencia respecto de la efectividad de distintas intervenciones es aún limitada. Las prácticas más efectivas se basan en el rol de los cuidadores principales para apoyar a los niños, animarlos pero no presionarlos a expresar sus emociones, entregar información que ayude a normalizar la situa- 
ción y responder a los miedos y las preguntas que surjan. Sin embargo, las autoras destacan que hay pocas intervenciones basadas en evidencia disponibles para los niños y que se debe reconocer también los efectos que tienen estos eventos en los cuidadores principales, porque ellos pueden impedir el reconocimiento de las necesidades emocionales de los niños en estas situaciones ${ }^{43}$.

Finalmente, una brecha relevante es la limitada evidencia sobre el impacto de los desastres en la salud mental de la población en países en desarrollo. Gran parte de la literatura revisada corresponde a datos obtenidos en naciones desarrolladas. Sin embargo, el 95\% de las muertes debido a desastres y los mayores impactos sociales y económicos se producen en el mundo en desarrollo. Al mismo tiempo la limitación de la investigación en países de mediano y bajo ingresos, esta acompañada de dificultades para implementar protocolos respetuosos de los derechos de sujetos que participan en investigación.

\section{El 27F en Chile y los niños}

En la zona afectada, en el momento del terremoto había más de 30.000 mujeres en gestación y unos 300.000 niños/as menores de 6 años bajo control en la red pública de salud ${ }^{44}$. Un total de más de 400.000 niños y niñas menores de 6 años se vieron directamente afectados por el evento. Hasta este momento, existe escasa literatura que pueda informar sobre los resultados inmediatos y posteriores al terremoto, tanto en la salud mental, como en la adaptación de los niños y sus familias.

Pese a la carencia de estudios publicados acerca del impacto directo del terremoto en los niños Chilenos, recientemente dos trabajos han logrado documentar impactos significativos de la exposición a un terremoto en el primer trimestre de la gestación, tanto en la edad gestacional al parto, en el peso al nacer ${ }^{45,46}$, como en la probabilidad de rotura prematura de membranas y restricción de crecimiento intrauterino ${ }^{47}$. Lo llamativo de estos hallazgos, es que ambos grupos de investigación llegaron de manera independiente a conclusiones simi- lares utilizando diferentes fuentes de datos de nacimiento y distintas metodologías para el análisis de los datos. Estos estudios utilizan como exposición el terremoto de Tarapacá del año 2005 y el de Concepción el año 2010 respectivamente. Ambos estudios son robustos en cuanto a los tamaños de su muestra y al control de variables relevantes para los resultados al parto.

Otro estudio, recientemente publicado, es el que utiliza los datos provenientes de la CASEN posterremoto ${ }^{48}$. La encuesta recolectó datos de 22.159 hogares, que corresponden a un subconjunto de la población entrevistada en la CASEN 2009. En tal sentido, estos datos son de carácter longitudinal puesto que se entrevista a un mismo grupo de personas el 2009 y 2010, y contiene información de resultados en salud mental inmediatamente después del terremoto utilizando la Escala de Trauma de Davidson $^{5,48}$. Los análisis reportados hasta ahora, se han centrado sólo en la descripción general sobre los síntomas de estrés postraumático en la población adulta, no en los menores de edad de los hogares entrevistados, y se desconoce las implicancias que podrían tener estos datos para la comprensión de diversas exposiciones producto del terremoto, y que pudieran impactar en la salud mental de los niños.

Por otra parte, datos recolectados por UNICEF un mes después del terremoto, incluyendo una muestra 369 niños de entre 12 y 15 años viviendo en las zonas afectadas, mostraron que el $90 \%$ de los niños podía reconocer algún efecto psicológico en si mismo producto del desastre; $37 \%$ se despertaba durante la noche, el $31 \%$ prefería no quedarse solo en su casa, $27 \%$ no quería estar lejos de sus padres, $21 \%$ tenía pesadillas y $17 \%$ necesitaba quedarse dormido con un adulto cerca. Además, prácticamente todos (97\%) se declaraban estar preocupados por las consecuencias del sismo, en especial por la gente que lo había perdido todo $(40 \%)$, pero también por las réplicas o incluso que ocurriera otro terremoto $(15 \%)$. Por otra parte, cuando se les preguntó a los niños sobre qué tan afectada había sido su ciudad y otras ciudades del país; todos los niños referían que otras ciudades del país habían sido más afectadas. En tanto que los niños/as de 
Talca y Concepción señalaban a sus ciudades como altamente afectadas, tanto como otras ciudades del país.

Respecto del impacto de las intervenciones implementadas en distintas zonas del país, sabemos muy poco. Principalmente debido a la gran cantidad de iniciativas espontáneas y poco documentadas implementadas por distintas Organizaciones No Gubernamentales (ONG), de la sociedad civil organizada, de grupos profesionales y del propio estado que implementó acciones preventivas en materia de salud mental, a través de los municipios y redes de proveedores de servicios sociales, de salud y educación, lo que hace muy difícil evaluar la efectividad de cada una de éstas intervenciones. Y como es común en situaciones de desastre, muy pocas se diseñaron con un plan de evaluación durante el período de respuesta inmediata. Un gran aporte a la comprensión de lo que sucedió en materia de primera ayuda psicológica, es el trabajo de Méndez y cols, quienes prontamente reportaron un mapeo de caracterización de las primeras intervenciones psicosociales realizadas en la Región del Maule ${ }^{49}$. Iniciativas de este tipo puede ayudar a comprender mejor qué prácticas son usadas y potencialmente evaluar estas intervenciones.

Por otra parte, la intervención implementada por Sommer y cols (2013, en este número $)^{50}$, incorporó distintos componentes orientados a intervenir en los casos clínicos y entregar herramientas a profesores. Si bien no se reportan resultados respecto a su efectividad, el tipo de intervención va en la línea de lo recomendado por organismos internacionales y estudios como los de LaGreca y Silverman (2009) ${ }^{43}$. Los autores reconocen la importancia de entregar atención a las personas que presentan sintomatología clínica y entregar herramientas a los cuidadores principales y personas cercanas del entorno del niño, quienes pueden ayudar a contener a los menores que se habían visto expuestos al terremoto.

El estudio de Bedregal y cols considera como variable central (2013, en este número) $)^{51}$ la salud mental y creencias sobre la crianza de 1.625 cuidadores de todo el país. Resalta de este, el que la prevalencia de TEPT reportada se ajusta bien a las estadísticas nacionales pre- vias y otros estudios poblacionales en el mundo. La información es tremendamente rica y puede considerarse que la recolección de los datos 6 meses después del terremoto, especialmente los exámenes al desarrollo del niño son un acierto desde el punto de vista metodológico.

Hasta donde nosotros sabemos, no existen otros trabajos publicados sobre efectos directos en la salud mental de los niños preescolares y escolares.

\section{Conclusiones}

La literatura internacional muestra de manera consistente que los niños expuestos a desastres naturales pueden presentar distintos trastornos de salud mental y que algunas condiciones, como exposición a otros estresores previos y otros posteriores como el desplazamiento, la relocalización, las pérdidas materiales y la salud mental de sus propios cuidadores, se asocian con mayores problemas de salud mental en niños.

Si bien la investigación en Chile es limitada y sabemos poco respecto a los niños y cómo se han visto afectados por el terremoto del 27 de febrero, los avances en los últimos años han sido significativos. En los últimos tres años ha habido un aumento importante en la investigación orientada a entender este problema y los artículos publicados en este número de la Revista Chilena de Pediatría son un excelente ejemplo de esto. Por otro lado, existe una preocupación cada vez mayor por sistematizar información respecto a intervenciones implementadas. En este sentido, el estudio de Méndez y cols, que buscó registrar y organizar la información disponible respecto de las intervenciones implementadas en la Región del Maule, es un ejemplo que debiera ser llevado a una mayor escala. El trabajo de Sommer y cols, es quizá uno de los únicos publicados hasta ahora y que puede permitir conocer como fue implementado un programa preventivo en la realidad. Sin embargo, dada la limitada cobertura del mismo no es posible establecer conclusiones respecto de su impacto ${ }^{50}$. Por otra parte, el estudio de Bedregal y 
cols, presentado aquí como un estudio transversal tiene enormes potencialidades en cuanto al seguimiento longitudinal de la población estudiada. Como parte de la evaluación del Programa de Apoyo al Desarrollo Biopsicosocial, del Sistema de Protección a la Infancia, Chile Crece Contigo (2010-2012), estos datos pueden permitir conocer en corto tiempo como la salud mental del cuidador enfrentado a una situación potencialmente traumática puede impactar el desarrollo y la salud mental de los niños. Aunque, los autores no reportan efectos sobre el desarrollo infantil en este corte transversal, creemos que las variaciones naturales en respuesta al estrés del terremoto al interior del país, tienen enormes potencialidades para comprender mecanismos de impacto del estrés en el desarrollo infantil ${ }^{51}$.

Una de las mayores brechas de información corresponde a lo sucedido con las familias que vivieron desplazadas y que se encuentran en reubicación tres años después del evento, un grupo que se encuentra en alto riesgo de presentar problemas de salud mental, debido a la exposición crónica a estresores derivados del desastre. Un trabajo importante, en este sentido es la alianza de trabajo entre la Universidad de Harvard y la Universidad de Concepción a través de la iniciativa "Recupera Chile" 52 , un programa basado en la comunidad y que busca la integralidad en el proceso de reconstrucción y recuperación de algunas localidades en la VIII región. Los resultados de esta intervención y su evaluación pueden contribuir a mejorar las prácticas y la planificación de los procesos de reconstrucción en el futuro.

También es importante notar que existe una variedad de trabajos, con muestras pequeñas, con diferentes instrumentos medición y limitaciones metodológicas para controlar sesgos, lo que da cuenta de una gran variación en la implementación de las intervenciones e investigación. Por esta razón, iniciativas como del nuevo Centro Nacional de Investigación para la Gestión Integrada de Desastres Naturales $(C I G I D E N)^{53}$, financiado por el Fondo de Investigación Avanzado en Áreas Prioritarias (FONDAP) y que es integrado por varias universidades espera transformarse en un catalizador de estas investigaciones y un repositorio de datos y estudios, para contribuir a potenciar la investigación multidisciplinaria sobre desastres en Chile.

Finalmente, los desafíos éticos de la investigación sobre desastres son enromes y claves para el avance de la investigación en esta área. No sólo se debe reconocer la importancia de revisiones expeditas de los comités de ética ${ }^{54}$, con el objeto de implementar los estudios lo más rápido posible. Es clave poner en el centro las múltiples necesidades y experiencias de las víctimas y protegerlas de las secuelas de la investigación no controlada. Al implementar estos estudios se debe tener especial preocupación por no interferir con la atención que reciben las personas en sus comunidades y con las redes de apoyo local. También se debe evitar el comunicar la participación en los estudios como una intervención que provee de ayuda, si este no se trata de un estudio de tratamiento ${ }^{55}$, dado que esto puede inhibir la búsqueda de ayuda posterior. Los procedimientos éticos deben también considerar si las personas que se encuentran más afectadas pueden tomar realmente una decisión informada y qué tipo de respuesta se puede ofrecer a aquellos a quienes se les detecta un trastorno de salud mental ${ }^{56}$.

En Chile queda mucho por avanzar en la implementación de consentimientos informados realmente descriptivos del proceso de participación en un estudio, sobre todo en el reconocer que no existen preguntas inocuas. Muchos cuestionarios y entrevistas estandarizadas o no, pueden tener efectos negativos tales como activar recuerdos traumáticos y traer malestar psicológico, lo que debe ser claro para los participantes de los estudios.

Una respuesta muy natural nace cuando hay un desastre y es que la gente quiere ayudar, sin embargo intervenciones diseñadas de un modo bien intencionado pero por equipos sin experiencia y sin relaciones de confianza con la población afectada pueden terminar en efectos nocivos. Las investigaciones pos-desastre deben adherir a los mismos estándares de toda investigación con seres humanos, y destinarse mayores recursos al estudio sobre la efectividad de las intervenciones preventivas o de tratamiento. Las investigaciones basadas en fuertes principios éticos en respuesta a un 
desastre, pueden ayudar a diferenciar qué sirve y qué no o incluso describir aquello que puede causar daño. Un ejemplo de ello, es que solo a través de la investigación en el tratamiento de niños con trauma severo se ha podido entender que las intervenciones basadas en que el niño o niña cuente la historia, o la repetición en varios contextos (juez, familia, escuela), tendría un efecto re-traumatizante.

Sabemos que los desastres naturales son eventos complejos, por su impredictibilidad y masividad en los impactos. Sin embargo, estos eventos son oportunidades para el desarrollo y el aprendizaje de los países. El 27 de febrero de 2010 quedara grabado en la mente de toda una generación. Aprender de esta experiencia es un deber que tenemos con los niños chilenos y otros miles de millones de niños que viven en naciones en desarrollo.

\section{Referencias}

1.- Fariña L, Opazo C, Vera P: Impactos ambientales del terremoto y tsunami en chile. Las réplicas ocultas del 27f. Fundación Terram en colaboración con la Unión Europea 2013.

2.- Madariaga $R$ : Sismicidad de Chile. Física de la tierra 1998; (10): 221.

3.- Moehle J, Riddell R, Boroschek R: The Mw 8.8 Chile Earthquake of February 27, 2010. Earthquake Engineering 2010. Retrieved from https://www.eeri.org/site/ images/eeri_newsletter/2010_pdf/Chile10_insert.pdf

4.- OIT: Proyecto impacto del terremoto sobre el empleo 2010. Desacargado de http://www.oitchile.cl/pdf/10-01. pdf

5.- Larrañaga O, Herrera R: Encuesta Post Terremoto: Principales resultados. Ministerio de Desarrollo Social 2010.

6.- Arbour M, Murray K, Arriet F, Moraga C, Cordero M: Lessons from the Chilean earthquake: how a human rights framework facilitates disaster response. Health and Human Rights 2011; 13 (1): e70-e81.

7.- Morris J, Van Ommeren M, Belfer M, Saxena S, Saraceno $B$ : Children and the Sphere standard on mental and social aspects of health. Disasters 2007; 31 (1): 71-90.

8.- Prinstein MJ, La Greca AM, Vernberg EM, Silverman WK: Children's Coping Assistance: How Parents, Teachers, and Friends Help Children Cope After a Natural Disaster. Journal of Clinical Child and Adolescent Psychology 1996; 25 (4): 463-75.
9.- Schoenbaum MM, Butler BB, Kataoka SS, et al: Promoting mental health recovery after hurricanes Katrina and Rita: what can be done at what cost. Archives of General Psychiatry 2009; 66 (8): 906-14.

10.- Vernberg EME, Silverman WKW, La Greca AMA, Prinstein MJM: Prediction of posttraumatic stress symptoms in children after hurricane Andrew. Journal of Abnormal Psychology 1996; 105 (2): 237-48.

11.- Kar N, Bastia BK: Post-traumatic stress disorder, depression and generalised anxiety disorder in adolescents after a natural disaster: a study of comorbidity. Clinical Practice and Epidemiology in Mental Health 2006; 2 (1): 17.

12.- Kronenberg ME, Hansel TC, Brennan AM, Osofsky, HJ, Osofsky JD, Lawrason B: Children of Katrina: lessons learned about postdisaster symptoms and recovery patterns. Child Development 2010; 81 (4): 1241-59.

13.- Mollica RF, Cardozo BL, Osofsky HJ, Raphael B, Ager $A$, Salama $P$ : Mental health in complex emergencies. Lancet 2004; 364 (9450): 2058-67.

14.- Norris FH, Friedman MJ, Watson PJ, Byrne CM, Díaz, E, Kaniasty K: 60,000 disaster victims speak: Part I. An empirical review of the empirical literature, 1981-2001. Psychiatry 2002; 65 (3): 207-39.

15.- Shannon MP, Lonigan CJ, Finch AJ, Taylor CM: Children exposed to disaster: I. Epidemiology of posttraumatic symptoms and symptom profiles. Jaac 1994; 33 (1): 80-93.

16.- La Greca AA, Silverman WKW, Vernberg EME, Prinstein MJM: Symptoms of posttraumatic stress in children after Hurricane Andrew: a prospective study. Journal of Consulting and Clinical Psychology 1996; 64 (4): 71223.

17.- Bonanno GA, Brewin CR, Kaniasty K, La Greca AM: Weighing the Costs of Disaster: Consequences, Risks, and Resilience in Individuals, Families, and Communities. Psychological Science in the Public Interest 2010; 11 (1): 1-49.

18.- Evans L: Children and Natural Disasters: A Primer for School Psychologists. School Psychology International 2006; 27 (1): 33-55.

19.- Díaz CA, Quintana GR, Vogel EH: Síntomas de depresión, ansiedad y estrés post-traumático en adolescentes siete meses después del terremoto del 27 de febrero de 2010 en Chile. Terapia Psicológica 2012; 30 (1): 37-43.

20.- Briceño AAM, Abufhele MM, Dávila DA, et al: Estrés postraumático en escolares a 8 meses del 27F. Rev Chil Pediatr 2013; 84 (1): 32-40.

21.- Cova F, Valdivia M, Rincón P, et al: Estrés postraumático en población infantojuvenil post 27F. Rev Chil 
Pediatr 2013; 84 (1): 22-31.

22.- Weissbecker I, Sephton SE, Martín MB, Simpson DM:

Psychological and physiological correlates of stress in children exposed to disaster: Current research and recommendations for intervention. Children, Youth and Environments 2008; 18 (1): 30-70.

23.- Bonanno GA, Mancini AD: The Human Capacity to Thrive in the Face of Potential Trauma. Pediatrics 2008; 121 (2): 369-75

24.- Catani C, Gewirtz AH, Wieling E, Schauer E, Elbert $T$, Neuner F: Tsunami, war, and cumulative risk in the lives of Sri Lankan schoolchildren. Child Development 2010; 81 (4): 1176-91.

25.- Shonkoff JPJ: Building a new biodevelopmental framework to guide the future of early childhood policy. Child Development 2010; 81 (1): 357-67.

26.- Abramson DM, Park YS, Stehling-Ariza T, Redlener I: Children as bellwethers of recovery: Dysfunctional systems and the effects of parents, households, and neighborhoods on serious emotional disturbance in children after Hurricane Katrina. Disaster Medicine and Public Health Preparedness 2010; 4 (Supplement 1): S17-27.

27.- Masten AS, Narayan AJ: Child Development in the Context of Disaster, War, and Terrorism: Pathways of Risk and Resilience. Annual Review of Psychology 2012; 63 (1): 227-57.

28.- Osofsky HJ, Osofsky JD, Kronenberg M, Brennan A, Hansel TC: Posttraumatic Stress Symptoms in Children After Hurricane Katrina: Predicting the Need for Mental Health Services. American Journal of Orthopsychiatry 2010; 79(2): 212-20

29.- Peek L, Morrissey B, Marlatt H: Disaster Hits Home: A Model of Displaced Family Adjustment After Hurricane Katrina. Journal of Family Issues 2011; 32 (10): 137196

30.- Araya R, Montgomery A, Rojas G, et al: Common mental disorders and the built environment in Santiago, Chile. The British Journal of Psychiatry : the Journal of Mental Science 2007; 190: 394-401.

31.- Vicente B, Barra F, Saldivia S, Kohn R, Rioseco P, Melipillán R: Prevalence of child and adolescent psychiatric disorders in Santiago, Chile: a community epidemiological study. Social Psychiatry and Psychiatric Epidemiology 2011: 47 (7): 1099-109.

32.- Vicente B, Saldivia S, la Barra de F, et al: Prevalence of child and adolescent mental disorders in Chile: a community epidemiological study. Journal of Child Psychology and Psychiatry 2012; 53 (10): 1026-35.

33.- Ministerio de Desarrollo Social: Infancia y Adolescencia en CASEN 2011. Presentación de resultados del estudio 2011. Desacargado de http://observatorio.ministeriodesarrollosocial.gob.cl/layout/doc/casen/publicaciones/2011/RESULTADOS_FINALES_INFANCIA.pdf

34.- Kieling C, Baker-Henningham H, Belfer $M$, et al: Child and adolescent mental health worldwide: evidence for action. The Lancet 2011; 378: 1515-25.

35.- Durkin ME: The emotional impact of post-disaster relocation. FMHI Publications 1987. Paper 11.

36.- Boyce WT, Ellis BJ: Biological sensitivity to context: I. An evolutionary-developmental theory of the origins and functions of stress reactivity. Development and Psychopathology 2005; 17 (2): 271-301.

37.- Loman MMM, Gunnar MRM: Early experience and the development of stress reactivity and regulation in children. Neuroscience and Biobehavioral Reviews 2010; 34 (6): 867-76.

38.- Shonkoff JP, Siegel BS, Dobbins MI, et al: Early Childhood Adversity, Toxic Stress, and the Role of the Pediatrician: Translating Developmental Science Into Lifelong Health. Pediatrics 2011; número: 224-231.

39.- Bonanno GA, Mancini AD: Beyond resilience and PTSD: Mapping the heterogeneity of responses to potential trauma. Psychological Trauma: Theory, Research, Practice, and Policy 2012; 4 (1): 74-83.

40.- Trickey D, Siddaway AP, Meiser-Stedman R, Serpell $L$, Field AP: A meta-analysis of risk factors for posttraumatic stress disorder in children and adolescents. Clinical Psychology Review 2012; 32 (2): 122-38.

41.- Bonanno GA, Westphal M, Mancini AD: Resilience to loss and potential trauma. Annual Review of Clinical Psychology 2011; 7: 511-35.

42.- Inter-Agency Standing Committee: IASC guidelines on mental health and psychosocial support in emergency settings. Inter-Agency Standing Committee. 2007.

43.- La Greca AM, Silverman WK: Treatment and prevention of posttraumatic stress reactions in children and adolescents exposed to disasters and terrorism: What is the evidence? Child Development Perspectives 2009; 3 (1): 4-10

44.- DEIS-MINSAL: Elaboración: Secretaría Ejecutiva Chile Crece Contigo Cobertura de controles de salud del niño(a) 2010. Ministerio de Salud.

45.- Torche F, Kleinhaus K: Prenatal stress, gestational age and secondary sex ratio: the sex-specific effects of exposure to a natural disaster in early pregnancy. Human Reproduction 2012; 27 (2): 558-67.

46.- Torche FF: The effect of maternal stress on birth outcomes: exploiting a natural experiment. Demography 2011; 48 (4): 1473-91.

47.- Oyarzo C, Bertoglia P, Avendaño R, et al: Adverse pe- 
rinatal outcomes after the February 27th 2010 Chilean earthquake. Journal of Maternal-Fetal and Neonatal Medicine 2012; 25 (10): 1868-73.

48.- Zubizarreta JR, Cerda M, Rosenbaum PR: Effect of the 2010 Chilean earthquake on posttraumatic stress: reducing sensitivity to unmeasured bias through study design. Epidemiology 2013 (Cambridge, Mass.) 24 (1): 79-87.

49.- Méndez MD, Leiva MC, Bustos CB, Ramos NA, Moyano-Díaz E: Mapa exploratorio de intervenciones psicosociales frente al terremoto del 27 de Febrero de 2010 en la zona centro-sur de Chile. Terapia Psicológica 2010; 28 (2): 193-202.

50.- Sommer AK, Abufhele MM, Briceño AAM, et al: Intervención de salud mental en niños expuestos a desastre natural. Rev Chil Pediatr 2013; 84 (1): 49-57.

51.- Bedregal GP, Camila Carvallo PC, Hernández LlV,
Prado RP: Estrés post-traumático post terremoto $27 \mathrm{~F}$ en cuidadores de niños preescolares. Factores asociados del cuidador, de la familia y la crianza. Rev Chil Pediatr 2013; 84 (1): 41-8.

52.- http://www.recuperachile.cl

53.- http://www.cigiden.cl

54.- Collogan LKL, Tuma FF, Dolan-Sewell RR, Borja SS, Fleischman ARA: Ethical issues pertaining to research in the aftermath of disaster. Journal of Traumatic Stress 2004; 17: 363-72.

55.- Collogan LKL, Tuma FF, Dolan-Sewell RR. Borja SS, Fleischman ARA: Ethical issues pertaining to research in the aftermath of disaster. Journal of Traumatic Stress 2004; 17: 363-72.

56.- Rosenstein DLD: Decision-making capacity and disaster research. Journal of Traumatic Stress 2004; 17 (5): 373 81. 